\title{
Etude théorique et expérimentale de la baisse de rendement des turbines Francis par cavitation à bulles
}

\author{
par Ch. Arn, Ph. Dupont, F. Avellan \\ IMHEF-EPFL, Lausanne
}

\begin{tabular}{|c|c|c|c|}
\hline & & OMENCLATURE & \\
\hline & $\mathrm{C}$ & Vitesse & {$[\mathrm{m} / \mathrm{s}]$} \\
\hline & $\mathrm{R}$ & Rayon & {$[\mathrm{m}]$} \\
\hline & $\mathrm{E}=\mathrm{gH}$ & Energie hydraulique massique & {$[J / \mathrm{kg}]$} \\
\hline I INTRODLCTION & $\mathrm{Q}$ & Débit-volume & {$\left[\mathrm{m}^{3} / \mathrm{s}\right]$} \\
\hline Dans le cas des lurbines Francis, la cavitation à bulles sćparécs & NPSE & Energie nelte à l'aspiration & {$[\mathrm{J} / \mathrm{kg}]$} \\
\hline $\begin{array}{l}\text { apparaît dans des domaines de fonctionnement proches du point } \\
\text { de meilleur rendement. La répartition de pression sur l'aubage de }\end{array}$ & $\mathrm{p}$ & Pression & {$\left[\mathrm{N} / \mathrm{m}^{2}\right]$} \\
\hline la roue avec des écoulements correspondant à de tels points de & pv & Pression de vapeur & {$\left[\mathrm{N} / \mathrm{m}^{2}\right]$} \\
\hline $\begin{array}{l}\text { fonctionnement possède un minimum près de la sorlic des aubes } \\
\text { puisque les angles d'incidence de l'écoulement au bord d'attaque } \\
\text { sont en général petits. On observe done dans certains cas une }\end{array}$ & $\chi_{2}=\left(\mathrm{p}_{\mathrm{re}}-\mathrm{p}_{r}\right) / \rho \mathrm{E}$ & $\begin{array}{l}\text { Coefficient local de cavitation } \\
\text { pour une turbinc }\end{array}$ & $1-1$ \\
\hline appartion oc cavitation à bulles localisée près de la sortie de l'au- & $c_{\mathrm{r}}=\left(\mathrm{p}-\mathrm{p}_{\mathrm{n}}\right) / \rho \mathrm{\rho}$ & Coefficicnt de pression & {$[-]$} \\
\hline ne. Ce type de cavitation est évidemment très sensible à la & $\varphi=\mathrm{Q} / \pi\left(\mathrm{j} \mathrm{R}^{3}\right.$ & Coefficicient de débit & {$[-]$} \\
\hline concentration de germes de cavitation contenus dans l'eau mais & $\psi=2 \mathrm{E} / \omega^{2} \mathrm{R}^{2}$ & Coefficient d'énergic & $1-1$ \\
\hline $\begin{array}{l}\text { niveau de pression général de l'installation. Pour cette raison, on } \\
\text { choisit cette altitude en fonction du développement de ce type de }\end{array}$ & 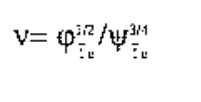 & Vitesse spécilique & {$[-]$} \\
\hline $\begin{array}{l}\text { cavitation au point de meilleur sendement. Les phénomènes phy- } \\
\text { siques provoquant ta baisse de rendement de la machine avec }\end{array}$ & $\rho$ & Masse volumique & {$\left[\mathrm{kg} / \mathrm{m}^{3}\right]$} \\
\hline I'apparition de cavitation à bulles ne son pas encore clairement & $\sigma=\mathrm{NPSE} / \mathrm{E}$ & Nombre de Thoma (Turbine) & {$[-]$} \\
\hline $\begin{array}{l}\text { déterminés. Des expériences menés avec un profil NACA } 009 \\
\text { installé sur une balance hydrodynamique }[9] \text { on montré que les }\end{array}$ & $\sigma=\frac{\left(p_{\text {ret }}-p_{v}\right)}{1 / 2 \rho C^{2}}$ & Coefficient de cavitation & {$[-]$} \\
\hline efforts d'origine hydraulique dépendent fortement du taux de & $\mathrm{k}$ & Numćro d'une ligne de maillage & {$[-]$} \\
\hline $\begin{array}{l}\text { germes ainsi que du coefficient de cavitation } \sigma \text {. On peut donc en } \\
\text { déduire que ce sont ces deux paramètres qui vont occasionner } \\
\text { une modilication de la distribution de pression sur l'aube en pré- }\end{array}$ & $*$ & $\begin{array}{l}\text { Valeur normalisée par rapport } \\
\text { à la valeur hors cavitation }\end{array}$ & \\
\hline
\end{tabular}

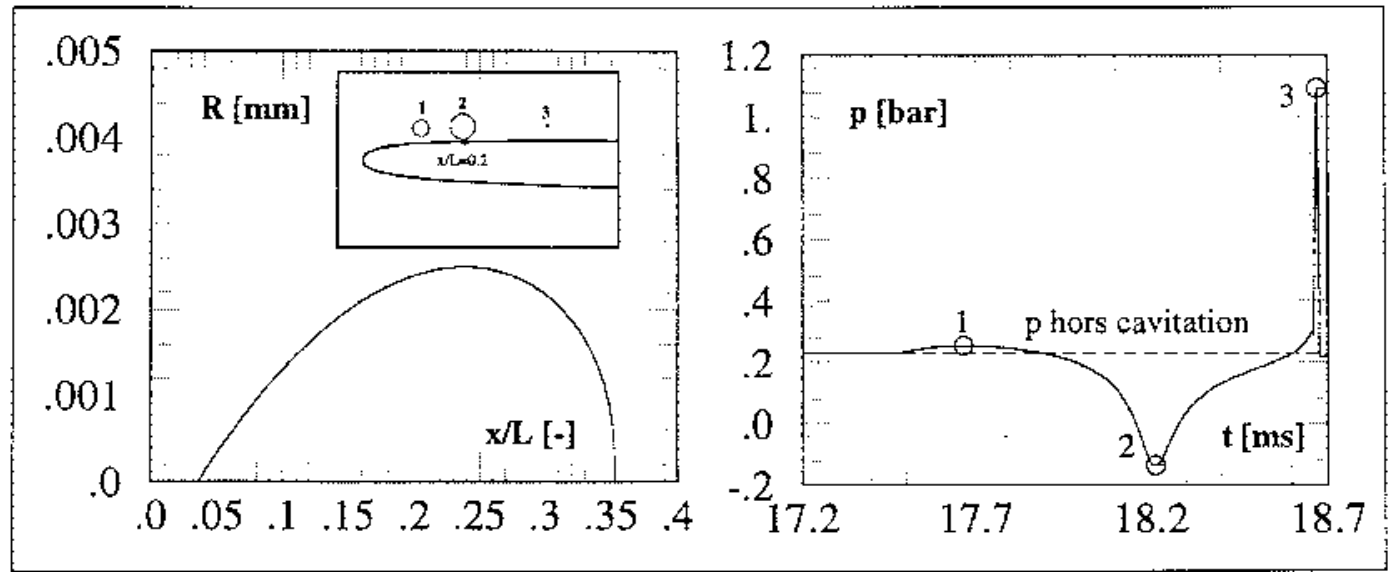

Figure 1: Evotution du rayon d'une bulle sphérique le long d'une ligne de courant à l'extrados d'un profil NACA 009. Pression générée au cours du temps en un point à $x / L=0,2$. 
sence de cavitation à bulles puisque la portance résulte de cette distribution. Line approche pour étudier l'influence de la cavitation à bulles est de considérer que la modification du champ de pression est principalement due a la dynamique de la bulle en évolution le long de l'aubage [7],[8],[10]. On peut observer un exemple de calcul de la pression générée en un poini du profil lors du passage d'une bulle sphérique à la figure 1.

Ces caículs de champ de pression basés sur la détermination d'un potcnticl d'une sphère cn expansion correspondent assez bien à la réalité, dans la mestirc ecpendart où les cavités sont peu déformées el resten! sphériques. L'amplitude des variations de pression est loutefois pus forte que celles que l'on peut mesurer [3]. mais ce type de modèle nous permet une bonne approche du phénomène physique. D'une manière générate, on remarque une légère surpression due au grossissement, puis une dépression plus marquéc avant te pic đê au collapse. En présence d'un développement de cavitation à bulles sphériques, on peut done assister à une légète baisse de la pression en moyenne. Pour des valeurs de o inféricures, la dimension des bulles devient plus grande que l'épaisseur de fluice contenant les germes aclifs, c'est-à-dirc l'épaisscur où la dépression est suftisante pour activer les germes de cavitation. Les bulles ont done tendance a s'aplatir jusqu'à former des hérnisphères [1]. Cette tendance est évidemment assez fortement liéc au nombre de Weber. Dans ec cas, la pression en paroi sous la butle est naturellement égale à la pression de vapeur. Si le taux de germes ou la taille des bulles sont sulfisants pour atteindre une saturation, 12 pression devient constante et égale à pv. On peut done caractériser trois zones différentes d'influence de la cavitation à bulles. La première est Ja zone où les bulles restent sphériques et la dynamique de la bulle génère les fluctuations de pression agissant sur la paroi. La seconde zone est la région où les bulles deviennent hémisphériques $\mathrm{cl}$ la dépression sous les bulles est limitée à la valeur de la pression de vapeur. La dernjère zone est une extension du dernicr cas avec un contact des bulles entre clles. II s'agil de la sacuration pour laquelle la pression devieni constante et égale à pv. L'objectî de cet article est de présenter les résultats $d^{+} u n e$ expérience menéc sur un profil bidimensionnel puis sur un modèle de turbine Francis pour vérifier ces différents points. L'expérience consiste en la mesure du champ de pression sur l'aubage pour différents régimes avec cavitation au moyen de capteurs de pression instationnaires embarqués.

\section{DISPOSITIF EXPERIMENTAL}

\subsection{Mesures sur le profil NACA 009}

Les essais sont menés sur le tunnel de cavitation à grande vitesse de l'IMHEF [5]. Le profil expérimental cst un profi] bidimensionmel NACA 009 de $100 \mathrm{~mm}$ de corde el $150 \mathrm{~mm}$ d'envergure tronqué à $90 \%$ de sa longucur. 18 capteurs de pression absolue sont distribués sur l'exirados du profil. Le montage des capteurs sur lc profil est ilitustré en figure 2. La gamme de mesure est de 0 à 100 bars. Chaque capteur possède sa propre alimentation et les sorties du signal de pression son amplifiées et riftrées séparément. L'acquisition ces signaux est elfectuée au moyen de deux cnregisireurs numériques d'une résolation de 12 bits. Le premier (Lecroy $8212 a$ ) p̣ernet l'acquisition simultanéc de 32 signaux à une fréquenec d'ćchantillonnage maximum de $5 \mathrm{kHz}$. Le second permet l'enregistrement simultané de 12 signaux à une fréquence maximum de $1 \mathrm{MHz}$ (3 modules Lecroy 6810). L'étaiontage statique de ces capleurs est mené avec l'aube monlée dans la section d'essai du tunnel de cavita-

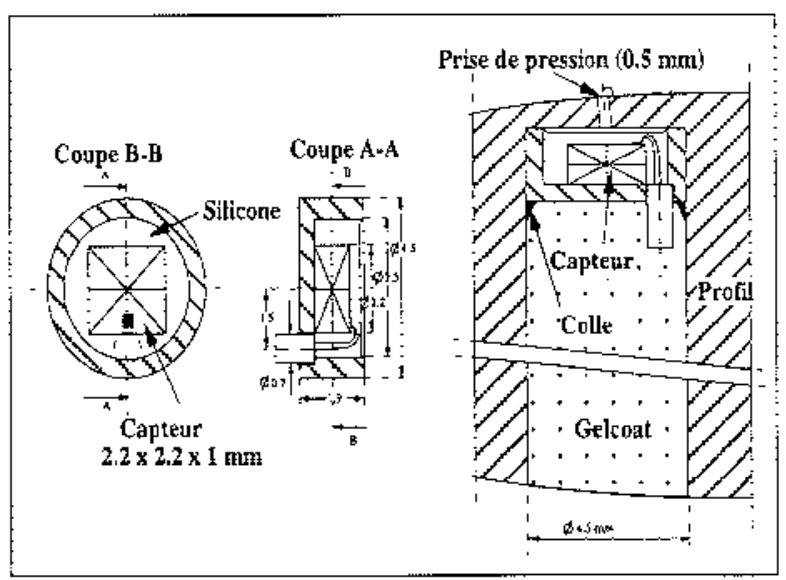

2. Montage des captcurs de pression.

tion en variant la pression statique de 0,3 a 10 bar. Line technique spéciale d'étalonnage dynamique a en outre été développéc pour ce genre de captetrs embarqués. On génère une impulsion de pression dans la veine d'essai grâce au collapse d'une bulle de vapeur générée au moyen d'une électrode. Les signail des capteurs à ćtalonner sont comparés au signai de pression d'un capteur de référence Kistler 601 monté également dans la section d'essai. En calculant les tonctions de iransfert et de cohérence entre les signaux des différents capteurs, on peut assurer is bande passante disponible de chacun des capteurs cmbarqués. Celle-ci va jusqu'à 15 ou $20 \mathrm{kHz}$ pour l'ensemble des capteurs ce ce iype.

Durant toutc la durée ćes expériences, un contrôle du taux de germes est effectué La cavitation à bulles séparées est en effet impossible sans la présence de ces derniers. On procède done à unc injection de germes dans le tunnel de cavitation ainsi qu'a un contrôle de la conceniration contenue dans l'eau d'essai. Les germes sont générés par une détcnte d'eau saturée en air dans une séric de modules d'injection [2]. En variant te nombre de ces mocules, on arrive à obtenir la concentration de germes de cavitation requise. La distribution obtenue est mesurée par un compteur de germes de cavitation [4] qui utilise l'effet Vertiri pour la déicction du grossissement explosif des getmes.

\section{i. 2.2. Mesures sur le modèle de roue Francis}

Les mesures sont eflectuées sur un modèle de urbine Francis de vitesse spécilique $v=0,33$, (nq=52). Le modèle est installé sur la plate-forme de recierche de l'IMHEF qui permet des chates d'essai allant jusquy'à $60 \mathrm{~m}$ à des débils-yolumes maximums de

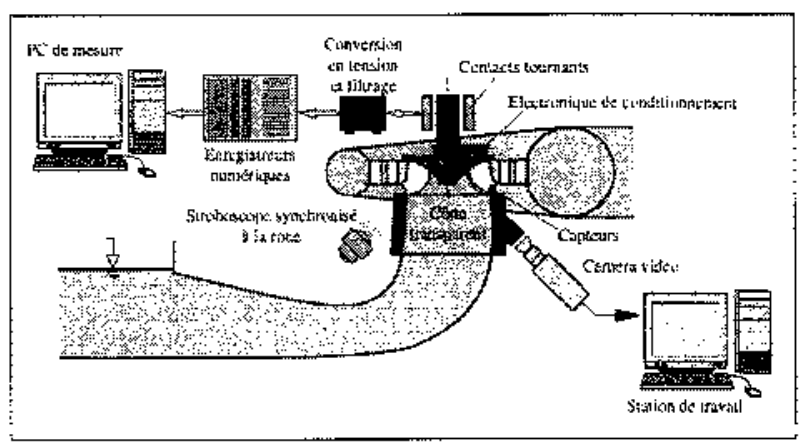

3. Chaine d'acquisition. 
$1,5 \mathrm{~m}^{3} / \mathrm{s}$. Les paramètres de mesure principaux comme le couple, le débil volume, la chulte, la viłesse de rolation, lá $1 \mathrm{em}$ pérature, etc. sont acquis en permanence pour délemminer des valeurs de la puissance et du rendement avec unc précision de mesure globale inféricure à $0,5 \%$. Deux aubes de la roue sont equipées avec chacune cing capteurs de type identique à ceux montés sur le profil bidimensiontel.

Jls sont positionnés près de la sortie el de la ceinture sur une section cylindrique de l'aube. Le système d'acquisition est le même que celui de l'expérience sur le profil bidimenșionnel, l'électronique de conditionnement mise à part. Cette dentrière est montée dans le plafond de la rouc. Elle compone deux amplificateurs à gain variable ainsi que deux multiplexeurs permettant la connexion à tous les captcurs embarqués. Les signaux de sortic sont convertis en fréquence pour minimiser les effets des perturbations électromagnétiques. Ja connexion du système de conversion en tension el d'acquisition avee les parties tournantes s'effectue au moyen d'un collecteur à contacts tournants. Les moyens d'enregistrenents sont également les mêrnes que pour l'expérience avec le profil bidimensionnel. La figure 3 pré. sente un schéma de la chaîne d'açuisition.

\section{D RÉSULTATS ET ANALYSES}

\subsection{Profil NACA 009}

Une expérience précédente menéc avec la même profil monté sur une balance hydrodymamique nous a permis de mesurer l'évolution du coelficient de portanec $\mathrm{c}$, en fonction du coefli. cient de cavitation $\sigma$. On obscrve sur les résultats obtenus pour une vitesse de $20 \mathrm{~m} / \mathrm{s}$ avec injection de germes de cavitation que la ponance augmente dès l'apparition de cavitation. Après un maximum atteint pour une valeur de $0 \div 0,35$, les valeurs chutent brusquement. Ces résultats sont reportés à ja fïgure 4.

L 'expérience décrite ici a done été menée pour mesurer la dis tribution de pression moyenne agissant sur l'aube dans les mêrnes conditions que les mesures de portance. Dans le cas d'une vitesse d'écoulement de $20 \mathrm{~m} / \mathrm{s}$ avec injection de germes de cavitation, les résultats obtenus sont préscnés à la figure $5 \mathrm{el}$ comparés avec la distribution de pression hors cavilation obtetue par ип calcul d'écoulement. Navier-. Stokes. Les images correspondant à chaque $\sigma$ sont ćgalement. présentćes sur lá figure. Pour la plus haute valeur de $\sigma$, correspondant donc à un régime sarıs cavitation, on remarque une tres bonne correspondance entre les calculs el les mesures. I.ors de l'apparition de cavita-

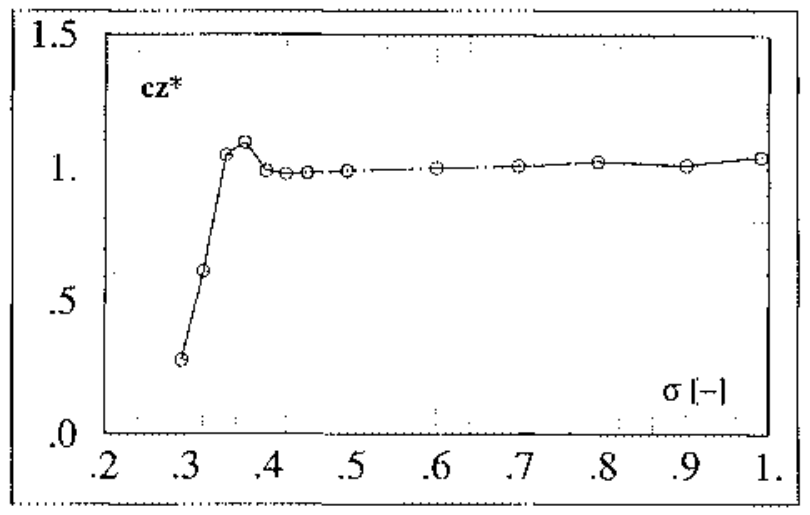

4. Coefficient de portance normalisé $c_{t}{ }^{*}$ en fonction du coefficient de cavitation $\sigma$. tion, une dépression apparâît dans la zone où le diamètre des bulles est maximum $(\sigma=0,4 \mathrm{cl} \times /,,-0,3)$. Dans ce cas, les bulles observées conservent une forme relalivement sphérique. Pour ○-0,375, ces bulles comntncncent à former des hémisphères et la dépression s'étend sur l'exirados du profil sans toutcfois atteindre la valeur limile de la pression de vapeur. Pour des valcurs encore inférieures de $\sigma$, les pressions mesurées sont égales à la pression de vapeur. On peut en effet remarquer sur les images correspondantes que la saturation est áteinle. I.a principale constatation que l'on puisse faire est que la baisse de portance par tapport à la valeur hors cavitation va apparaître Jorsque la pression de vapeur va limiler lá dépression. Cela va être illustré par le passage de la valeur de $\sigma$ au niveau du platcau de pression de la distribution sans cavitation. La valeur de ce platcau correspond à $c_{r}=-0,32$ et on remarque sur la figure 4 que c'cst pour une valcur relativement iclentique que l'on observe la valeur maximum de la portance. On peut encore ajouter que le comportement du profil en présence de cavitation à bulles va dépendre essentiellement du lieu où lá pression cst minimum sur l'aubc. Dans le cas où le $c_{n, m i:}$ est situé près du bord de fuite, ce qui correspond à de très fähles angles d'incidence, scule une baisse de partance va apparaître lorsque le développoment de

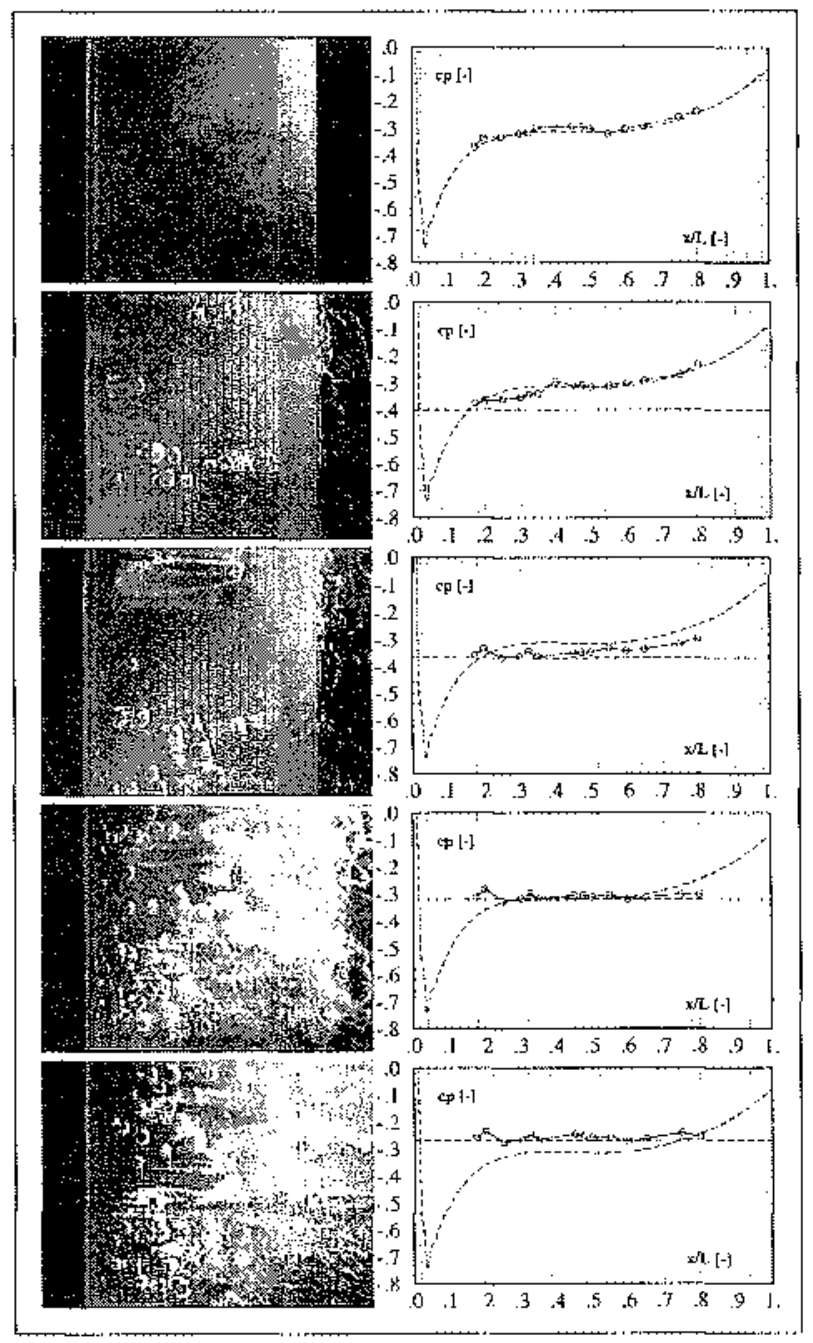

5. Distributions de pression mesurées et calculées hors cavitation à l'extrados du profil N $\mathrm{ACA} 009$ pour les valeurs de $\sigma$ suivantes : $1.0,0.4,0.375,0.325,0.275$. (De haut en bas). la ligne interrompue représente la valcur de $-\sigma$. 
Cavitation à bulles va entraîner une limitation de la pression à la valeur de la pression de vapeur. Dans le cas contraire oì le $\mathrm{c}_{\text {n.min }}$ est situé près du bord d'attaque, le développement de cavitation à billtes dans une zone de pression plus élevée que la pression de vapeur va posvoir augmenter la portance en permettant une dépression accruc. La baisse de portance ne va apparâ̂tre que lorsque le $\sigma$ sera assez bas pour que la présence de vapeur limite la dépression sur l'extrados.

\subsection{La roue Francis}

Généralement, la distribution de pression à l'extrados dè 'aubage d'une turoine Francis au point çe meilleur rendement correspond au premier des deux cas que nous avons décrits dans le paragraphe précćdent. L'angle d'inciòence ce l'écoulement est faible et la pression minimum est situće près de la soric de l'aubage. Les cssais menés sur le modèle on! couvert ce même point de fonctionnement pour trois chutes d'essai différcnics, $10 \mathrm{~m}$, $15 \mathrm{~m}$ et $20 \mathrm{~m}$. La répartition correspondante du coefficient de pression calculée à l'extrados des aubes cans lc cas d'une chutc de $15 \mathrm{~m}$ cst présentéc à la figure 6 .

On peul y observer que le minimum de pression est silué à la sortie de l'aubage pour chaque ligne de maillage de calcul sauf dans le zone proche de la ceinure $(k=19)$. D'après les anaiyses des mesures sur le profil bidimensionnel, le rendersent doit donc sculement décroître avec le coefficient de cavitation dès l'apparition de cavitation à bulles. Remarquons cn ortre que la torchc, pourtant assez sensibic au taux de germes, ne va avoir aucun effet puisqu'elle n'existe quasiment pas à ce point de fonctionnement. Rappelons que le coefficient de cavilation utitise habi. tuellement dans le cas des turbines est le nombre de Thoma $\sigma=$ NPSE $/$. Les courbes de cavitation obtenies lors des essais sont itlustrées en figure 7.

Les vateurs de rendement indiquées sont des valeurs relatives par rapport au rendement hors cavitation. On peut déterminer 12 valeur de $\sigma_{*}$ qui corsespond à la plus haute valeur ou nombre de Thoma oì le rendement est cncorc affecté. Cette valeur est la même dans le cas des trois différentes chites d'essai et vaut 0,068 . On peut done en déduire les valeurs du coefficient local de cavitation $\chi_{\mathrm{m}}$. La valeur obtenue pour la chute de $15 \mathrm{~m}$ est dc 0,022 . Ce résultat est très intéressant si l'on tente unc comparaison avec les coefficients de pression calcules. On remarque cn effel que la chute de rendement apparaît lorsquic l'cascmble de la sortie de ''asbage a une valeur de $c p$ inféricurc à $-\chi_{\mathrm{I}: \mathrm{p}}=-0,022$. Cela correspond déjà à un importani développement de cavita-

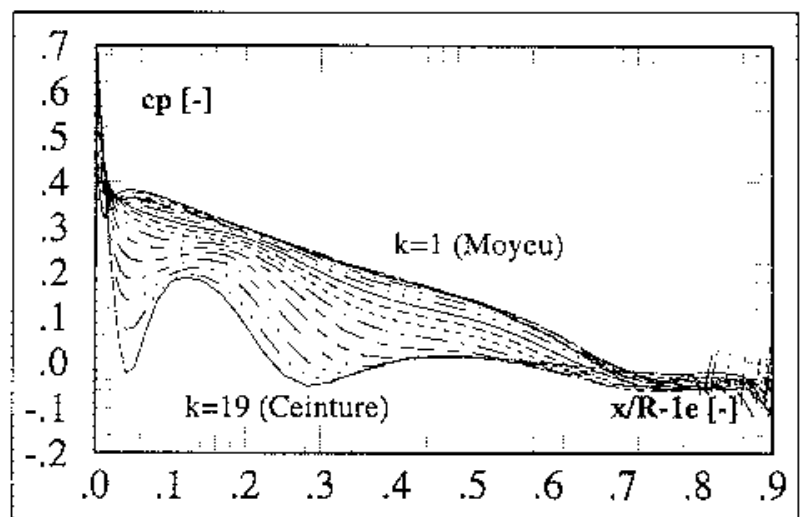

6. Résultats d'un calcul Navier-Stokes au point de meilleur rendement. La pression de référence est la pression de sortie du domaine de calcul.

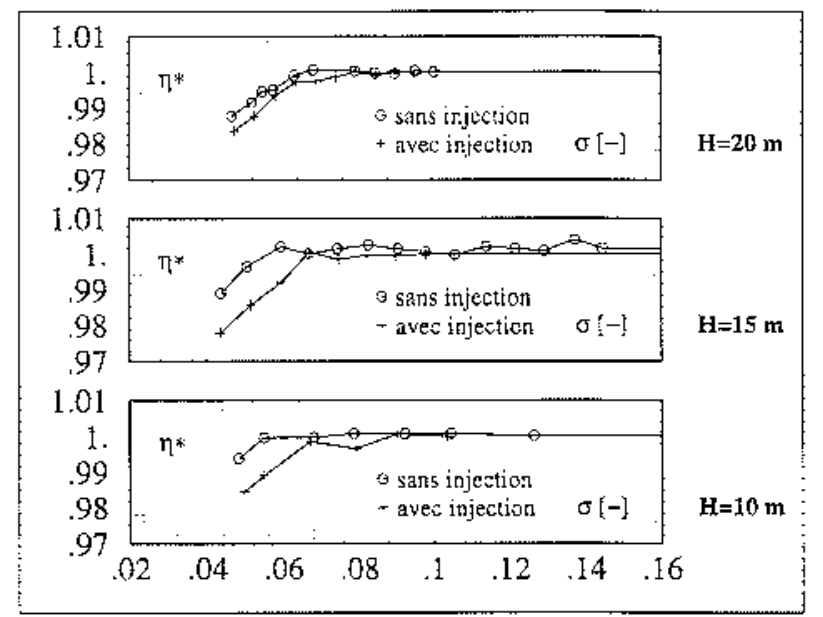

7. Courbes $\sigma-\eta \eta^{\dagger}$ mesurées lors des essais sur modèle de turbine Francis.

tion de sortie. On pest noter également que les premières bulles apparaissent pour un $\sigma$ valant 0,085 . On peut déterminer la valeur du nombre de Thoma nécessairc pour que la pression de vapeur soit atteinte au point de pression minimum de l'aubage en se basant sur les résultats du calcul d'écoulement. On trouve une valeur de $\sigma$ valant 0,11 . On peut donc consiater qu'il existe un certain retard de la baisse de rendement par rapport au moment où la pression de vapeur est atteinte sur l'aubege. Le développement de cavitation à bulles doit donc être déjà bien établi avant que le rendement ne vienne ì chuter. Comme dans le cas du profil bidimensionnel, on peut tracer les résultats des mesures de pression avec la distribution calcuiée sur la ligne de maillage correspondant à la posilion des capteurs. La figure 8 illustre ces résultats qui nous montrent, tout comme dans le cas cu proill bidimensionnel, une bonne concordance entre les cal-

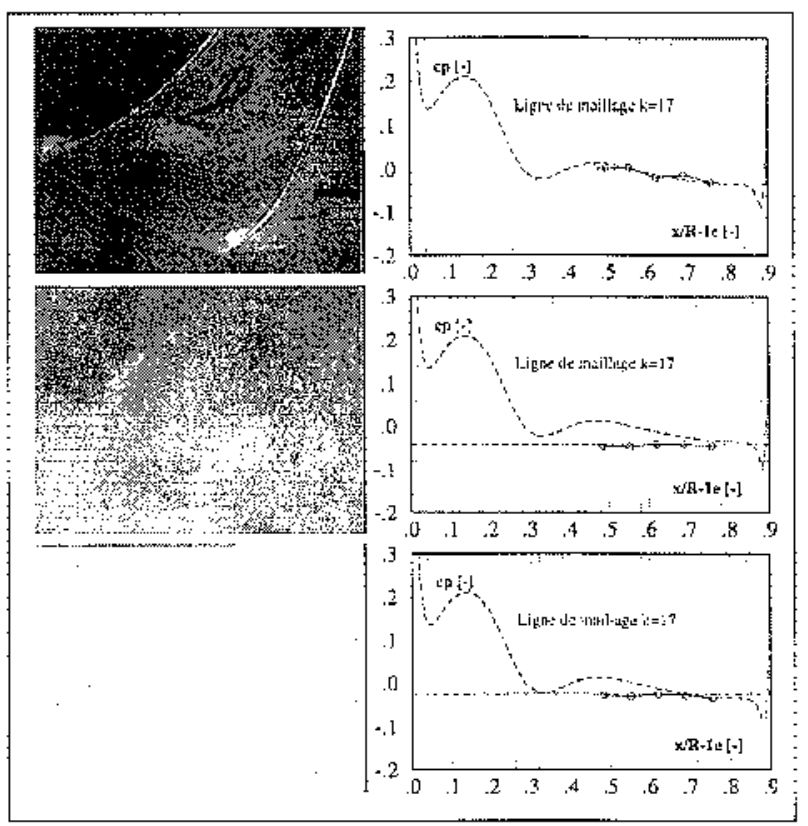

8. Distribution de pression mesurée ef calculée sur Ie fillet $k=17$ de l'aube du modèle de la roue Francis pour les valeurs de $\sigma$ suivantes: $0.43,0.07,0.06 . H=15 \mathrm{~m}$. La ligne horizontate interrompue représente la valeur de $-\chi_{1}$. 
culs et les mesures dans le cas hors cavitation. Pour les mesures à des valeurs de $\sigma$ inférieures, la distribution de pression va être modifiéc par le développenent de cavitation à bulles de maniète iderntique à te que l'on a pu mesurer sur le profil bidimen sionnel. On remarque une dépression jusqu'à la pression de vapeur due à un développement de cavitation à bulles provoqué par le pic de dépression localisé à l'amont des capteurs. Ce pic I'cxistant que près de la ceinture, le couple global va toul de même baisser sans générer une augmentation du rendement hydraulique.

\section{CONCLUSION}

Sur la base de ces deux expériences, nous avons pu mieux décrire les effets de la cavitation à bulles sćparées sur les performances d'une turbine Francis. La baisse de rendement apparâft principalement lorsque la saturation de cavitation est alteinte. Cette saluration provoque une modification de la distribution de pression cn linitant le champ de pression à la valcur de la pression de vapeur toul comme dans le cas de la cavitation à poche. Les performances de la machine ne sont pas fortement infuencécs par la partic de la machine où les bulles restent sphériques el le champ de pression modifić par lá dynamique propre des bulles de vapeur. L'érnission acoustique provoquée par l'ex. plosion et le collapse des bulles est à l'origine d'un certain nombre d'autres problèmes qui justifient l'étude du charnp de pression acoustique autour des bulles de cavitation. Cependant, dans l'optique d'obtenir une modélisation de la distribution de pression modifićc par la présence de cavitation à bulles, il cst nécessaire de déterminer les partics de l'aubage où les bulles vont rester sphériques, devenir hémisphériques el où la saturation de cavitation à bulles va être alteinte. I, a localisation de ces différentes régions dépend évidemment du taux de germes de cavitation et de point de fonclionnement de la machine. C"est la raison pour laquelle l'élude de l'évolution des bulles près d'unc paroi solide dans un champ de pression variable est importante dans le but de caractériser l'altération des performances d'une iurbomachine hydraulique. I.à voie à suivrc pour celte caractérisation dans le cas de la baisse de rendement due au développernent de cavitation à bulles est cortainement l'étude d'un modèle efficace de l'évolution d'une bulle dans une rone incluant sa déformation ainsi que le cas de la saturation. Les effets des différents types de bulles étant assez bien đécrits, c'est maintenant la connaissance de là répartition de ces différents types de développenent de cavitation dans la roue qui va rendre possible la prédiction de la baisse de rendement des lurbines Francis due au développement de cavitation à bulles séparées.

\section{REMERCIEMENTS}

Les auteurs tiennent particulièrement à remercicr les mernhres du groupe de recherche en cavitation de l'IMHEF ainsi que son personnel technique. Ces recherches ont ćté possible grâce au soutien financier du NEFF" "Nationalcr Energie Forschung Fonds" at du PSFI. "Fonds Suisses pour Projets et Eludes de l'Economie Electrique".

\section{RÉFÉRENCES}

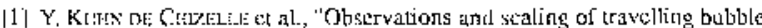
cavilatiota".J. I'luid. Mech., 293:99-126, 1995

[2] C. Brasu ct al., "Ihe IMHEF systern for cavilation mucfei injection." Sao Paolo, 1992. AIRII

[3] Cit. Akx ct al., "IExperimental and theoretical study of the 2t blade lift allecratien by traveling bubble cavitation." ASMit, August 1945

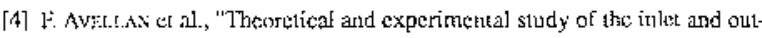

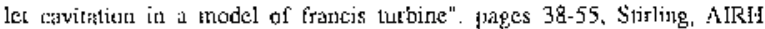
August 1984.

15. F. Avl1, AN cl al., "A new bigh s]eed cavitation lufuel for cavitation studies in hydraulic machintery." volume 57, paises 49 60. ASMli, 1987

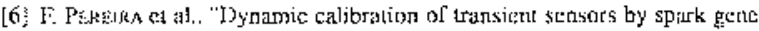
rated cavity." Symposium of Bubble 19ynamic and [nterface Pheronurna. Septernber 1993.

[7] J.e. Frave et al. "La Cavitation, Mécarismes physigurs a asplects jndustriels". Presses Lniversitaires de Grenoble, 1995.

[8] S. Ki,Mak et al., "A study of pressure puises generated by tratueliteg bubb]c Cavifation." 1. Hluid. Mech., 225:541 -564, 1993.

[9] B. GuNor(y.. "Lois de Sirnilitude dans les r̂ssais de Cavitation des ?urbines Francis." PhD thesis, EPFl, 1991

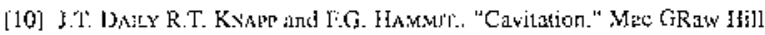
New York, $19 \%$ 